\title{
Associação entre capital social e bullying em adolescentes de 15 a 19 anos: relações entre $o$ ambiente escolar e social
}

\author{
Association between social capital and bullying among adolescents \\ aged between 15 and 19: relations between the school and social \\ environment
}

Andressa Reisen (https://orcid.org/0000-0003-3916-462X) ${ }^{1}$

Franciele Marabotti Costa Leite (https://orcid.org/0000-0002-6171-6972) 1,2

Edson Theodoro dos Santos Neto (https://orcid.org/0000-0002-7351-7719) 1,3
${ }^{1}$ Programa de PósGraduação em Saúde

Coletiva, Universidade Federal do Espírito Santo. Av. Marechal Campos 1468, Bonfim. 29047-105 Vitória ES Brasil. areisen@hotmail.com ${ }^{2}$ Departamento de Enfermagem, Universidade Federal do Espírito Santo. Vitória ES Brasil. ${ }^{3}$ Departamento de Medicina Social, Universidade Federal do Espírito Santo. Vitória ES Brasil.
Abstract The scope of this study was to analy$z e$ the association between social capital and bullying among adolescents aged 15 to 19 years, in high schools in the Greater Vitória Metropolitan Area in the State of Espirito Santo, Brazil. A crosssectional epidemiological survey was carried out at the school base, with a sample of 2293 students, stratified by municipality of school location. Descriptive and inferential statistics were performed using two instruments: Integrated Questionnaire to Measure Social Capital of the World Bank and adapted versions of the Olweus Bully/Victim Questionnaire. The results showed that victims of bullying were more likely to present low level of cognitive social capital ( $p=0.001 ; O R=1.9$, $95 \% C I=1.29-2.68)$, underlying $(p=0.002 ; \mathrm{OR}$ $=1.7,95 \% C I=1.20-2.38)$ and total $(p<0.001$; $O R=1.80,95 \% C I=1.32-2.59)$. Bullying offenders were associated with low levels of cognitive social capital $(p<0.001 ; O R=3.2,95 \% C I=$ 2.34-4.44) and total ( $p=0.042 ; O R=1.7,95 \% C I$ $=1.24-2.27$ ). High levels of social capital are related to the reduction of bullying and victimization behaviors. Therefore, healthy social relationships should be promoted in the spaces of mutual coexistence of adolescents to stimulate other positive reactions in school environments.

Key words Social capital, Social behavior, Bullying, Adolescent, Violence
Resumo O objetivo deste estudo foi analisar a associação entre capital social e bullying em adolescentes de 15 a 19 anos em escolas do ensino médio na Região Metropolitana da Grande Vitória, Espírito Santo, Brasil. Foi realizado um inquérito epidemiológico seccional de base escolar, com amostra formada por 2.293 estudantes, estratificada por município de localização escolar. Foram executadas estatísticas descritivas e inferenciais a partir de dois instrumentos: o "Questionário integrado para medir capital social do Banco Mundial" e o "Olweus bully/victim questionnaire", em versóes adaptadas. Os resultados demostraram que as vitimas de bullying tiveram maiores chances de apresentar baixo nivel de capital social cognitivo $(p=0,001$; OR $=1,9$; IC 95\% $=1,29$ $2,68)$, subjacente $(p=0,002 ;$ OR $=1,7 ;$ IC $95 \%=$ $1,20-2,38)$ e total $(p<0,001 ;$ OR $=1,80$; IC 95\% $=1,32-2,59)$. Os agressores de bullying foram associados a baixos níveis de capital social cognitivo $(p<0,001 ; O R=3,2 ;$ IC 95\% = 2,34-4,44) e total $(p=0,042 ;$ OR $=1,7 ;$ IC 95\% = 1,24-2,27). Elevados níveis de capital social estão relacionados à redução dos comportamentos de vitimização e de agressão por bullying. Portanto, deve-se promover relações sociais saudáveis nos espaços de convivência comum dos adolescentes para estimular outras reações positivas nos ambientes escolares.

Palavras-chave Capital social, Comportamento social, Bullying, Adolescente, Violência 


\section{Introdução}

O capital social se distingue de outras formas de capital, como o físico, que inclui recursos materiais e financeiros, e o humano, que incorpora a escolaridade e os conhecimentos, visto que está situado nas relações interpessoais ${ }^{1}$. Coleman ${ }^{2} \mathrm{o}$ define como a reciprocidade nas relações sociais, enquanto Putnam ${ }^{3}$ se refere às normas e redes formais e informais da estrutura social que habilitam os indivíduos a agirem juntos a fim de alcançar objetivos comuns. Portes ${ }^{4}$, por sua vez, refere-se aos recursos, na forma de informações, ideias e apoios, que os sujeitos são capazes de buscar em virtude de suas relações com as pessoas e que são acessíveis somente dentro e por meio das relações sociais.

Embora as definições apresentem alguma distinção, o capital social está diretamente conectado ao valor das relações sociais e fundamenta-se na maneira como essas relações se estabelecem. Caracteriza-se pelas formas que os membros de uma comunidade interagem e envolve, entre outros aspectos, as redes de relações entre grupos formais e informais de pessoas, cooperação, coesão social, confiança interpessoal, reciprocidade, troca de apoio social e comunicação, como bens inalienáveis das comunidades, o que torna o capital social um bem coletivo e uma propriedade de grupo ${ }^{1,5,6}$.

Segundo Grootaert e colaboradores (2003) o capital social é composto pelos seguintes indicadores: estrutural, cognitivo e subjacente. Os indicadores estrutural e cognitivo formam a base do capital social, enquanto o indicador de capital social subjacente se relaciona aos meios pelos quais o capital social opera. O capital social evidencia que formas e relações não-monetárias podem ser importantes fontes de benefícios provenientes de laços ou estruturas sociais ${ }^{3,7,8}$.

Resultados científicos associados ao capital social demonstram que níveis crescentes de engajamento apontam para a redução da exclusão social e aumentam e aprimoram o padrão de vida comunitária, promovendo uma sociedade mais saudável e menos violenta ${ }^{6,9}$. Vizinhanças com maior capital social vêm sendo associadas a menores percentuais de violência urbana ${ }^{6}$. Redes de apoio social surgem relacionadas a melhores resultados de saúde mental, a menos comportamentos problemáticos e à maior promoção da saúde. E o apoio estrutural de escolas e bairros com altos níveis de capital social vem sendo vinculado a melhores resultados de saúde e bem-es$\operatorname{tar}^{10}$. Na saúde coletiva, o capital social vem sen- do associado a aspectos da determinação social da saúde ${ }^{11}$.

Embora os estudos sobre o tema venham sendo majoritariamente executados com populações adultas, entre adolescentes o capital social tem sido relacionado a comportamentos de bullying, indicando que níveis mais elevados estão relacionados à redução da ocorrência do bullying ${ }^{9,12}$, o que revela a importância do capital social também dentro dos ambientes escolares e o seu potencial impacto na convivência escolar diária de crianças e adolescentes.

O bullying é um comportamento recorrente nos ambientes escolares e atinge populações estudantis no mundo e também no Brasil. Caracteriza-se pela repetitividade, intencionalidade e desigualdade de poder entre vítimas e agressores $^{13-15}$. Estudos ${ }^{13,16-18}$ demonstram de forma concreta os efeitos deletérios do envolvimento em bullying. Efeitos que incluem risco de danos físicos e psicológicos permanentes, que podem ser transportados para a vida adulta, até mesmo por aqueles que vivenciam o bullying de forma indireta $^{13-16}$, devendo ser combatido como uma via prática de promoção de saúde e prevenção de doenças.

Pesquisas brasileiras sobre o tema têm revelado prevalências preocupantes do comportamento $^{13,18,19}$. A Associação Brasileira Multiprofissional de Proteção à Infância e à Adolescência, em estudo pioneiro envolvendo mais de cinco mil estudantes no Rio de Janeiro (RJ) entre os anos de 2002 e 2003 , revelou que $40,5 \%$ dos estudantes admitiram estar diretamente envolvidos em atos de bullying ${ }^{13}$. Enquanto outra pesquisa, conduzida em Salvador (BA) em 2014 e 2015, apontou prevalência superior a $40 \%$ para a agressão e $55,2 \%$ para a vitimização de bullying ${ }^{19}$. Já estudo de 2015, empreendido com dados provenientes da Pesquisa Nacional do Escolar, revelou prática de bullying por 19,8\% dos estudantes avaliados ${ }^{18}$.

As evidências científicas das análises da relação entre capital social e comportamentos de bullying no cenário internacional indicam que o primeiro influencia tais comportamentos, repercutindo nos espaços de convivência estudantis $^{8,9,20,21}$. Os adolescentes que têm vínculos consistentes com a escola, com os professores e que recebem apoio dos colegas e dos amigos próximos, estão relacionados a menor probabilidade de envolvimento em bullying $8,9,20,21$. Indivíduos que possuem capital social mais elevado estão associados a maiores níveis de confiança e senso de justiça ${ }^{3}$ e a laços sociais mais fortes, resultando em redução do comportamento agressivo dentro 
das instituições escolares ${ }^{8,9,22}$. No entanto, o reduzido número de pesquisas que exploram essas relações revela a carência de estudos brasileiros e latino-americanos ${ }^{7,17}$ sobre a temática, embora estudos executados no eixo norte-americano-europeu venham demostrando a potencial ligação entre esses domínios ${ }^{8,9,10,20,22}$.

Sob os aspectos apresentados, este estudo propõe analisar a associação entre capital social (variável independente) e comportamentos de bullying (variável dependente) em adolescentes de 15 a 19 anos matriculados em escolas do ensino médio na Região Metropolitana da Grande Vitória, no Espírito Santo (RMGV-ES), Brasil.

\section{Métodos}

\section{Desenho do estudo}

Trata-se de um inquérito epidemiológico seccional, de base escolar, conduzido entre os anos de 2016 e 2017 com uma amostra de 2.293 estudantes de 15 a 19 anos da rede de ensino médio da RMGV-ES. Essa região abriga aproximadamente 1,6 milhão de habitantes, o que representa $48 \%$ da população do Espírito Santo, e cerca de 148 mil adolescentes de 15 a 19 anos $^{23}$. Segundo informações da Secretaria Estadual de Educação do Espírito Santo (2014), a RMGV-ES tinha 168 escolas de ensino médio e 65.763 estudantes regularmente matriculados. $\mathrm{O}$ ensino médio concentra os estudantes de 15 a 19 anos no Brasil (Lei 9.394/1996), faixa etária que integra este estudo. $\mathrm{O}$ enfoque em tal grupo populacional deu-se em razão da limitada disponibilidade de estudos científicos sobre os temas avaliados para o grupo etário na RMGV-ES. Os dados utilizados são provenientes da pesquisa "Vigilância de fatores de risco para doenças e agravos em adolescentes de 15 a 19 anos na RMGV-ES", cujo objetivo mensurar a exposição dos adolescentes a diferentes comportamentos de risco, doenças e agravos que podem afetar o desenvolvimento pleno e impactar a saúde física e mental.

\section{Critérios de inclusão e exclusão}

Foram elegíveis para este estudo todos os estudantes regularmente matriculados nas redes de ensino médio pública ou privada da RMGV-ES, dos períodos matutino e vespertino, com idade entre 15 e 19 anos, que não possuíssem qualquer tipo de necessidade especial, física ou mental, que impedisse sua participação ativa no estudo e o preenchimento do formulário eletrônico em notebook de forma individual. Foram incluídos todos os que concordaram em participar, entregaram os termos de consentimento e/ou termos de assentimento adequadamente assinados e que responderam de maneira efetiva às questões constantes do instrumento de pesquisa. Foram distribuídos 8.111 termos, com taxa de adesão de $30 \%$.

\section{Procedimentos amostrais e coleta de dados [}

A amostra foi estratificada por município, sendo calculadas cotas amostrais municipais correspondentes à distribuição percentual de estudantes matriculados nas escolas de ensino médio por município da RMGV-ES. A proporção amostral obtida foi muito próxima à amostra calcula$\mathrm{da}^{24}$ (Figura 1).

Considerando as proporções municipais inicialmente definidas, calculou-se o número de estudantes a ser entrevistado nos municípios da RMGV-ES. Todas as escolas que ofertavam ensino médio na RMGV-ES em 2014 foram enumeradas de forma sequencial, segundo o município de localização. A seleção amostral aleatória simples das escolas foi executada utilizando o programa BioEstat, versão 5.4, conforme a proporção até completar as cotas amostrais de cada município. Participaram do estudo 2.293 adolescentes escolares. A coleta de dados foi executada em 54 escolas, sendo 43 públicas e 11 privadas, e conduzida por pesquisadores previamente treinados, por meio de entrevista estruturada fechada em turmas selecionadas no horário das aulas. A leitura coletiva (em voz alta) de cada questão do instrumento, disponibilizado em forma de questionário eletrônico, foi conduzida pelo entrevistador responsável, e o preenchimento individual foi realizado pelos estudantes utilizando notebooks, concomitantemente à leitura. Um software específico para a realização da coleta de dados foi desenvolvido, permitindo a coleta de modo on-line e off-line.

\section{Instrumentos e variáveis}

\section{Características sociodemográficas}

Neste estudo foram incluídas as seguintes variáveis: idade (15 a 19 anos), sexo (masculino, feminino), raça-cor (branca, preta, parda, amarela, indígena), ano do ensino médio (primeiro, segundo, terceiro/quarto anos), turno de estudo (matutino, vespertino), rede de ensino (pública, privada), trabalho remunerado do adolescente 
(não, sim), anos de residência no endereço (até dez anos, acima de dez anos), saneamento básico (não, sim), número de moradores no domicílio (até cinco pessoas, mais de cinco pessoas), divórcio parental (não, sim), escolaridade do chefe da família (fundamental, médio, superior) e renda familiar total (até um salário mínimo R\$ 954,00, mais de um até três salários mínimos, mais de três até dez, mais de dez salários mínimos).

\section{Capital social}

Para avaliar o capital social, foi utilizada a versão curta do "Questionário integrado para medir capital social” (QI-MCS) do Banco Mundial $^{5}$, composto por seis dimensões que permitem a criação dos indicadores de capital social: estrutural (grupos e redes), cognitivo (confiança e solidariedade) e subjacente (ação coletiva e cooperação). Foi adotada uma classificação alternativa, a partir de Grootaert et al. ${ }^{5}$, para o indicador de capital social subjacente (CSS), visto que foram adicionadas a esse indicador as dimensões "informação e comunicação", "coesão e inclusão social" e "empowerment e ação política", a fim de captar o CSS de forma mais detalhada.

Todos os itens formadores do instrumento (indicador de capital social estrutural - CSE, composto por nove itens, capital social cognitivo - CSC, com sete itens, e CSS, com 17 itens) tiveram suas opções de resposta recodificadas em valores que variaram entre 0 e 10 , em que zero referiu-se à menor disponibilidade de capital social, e 10, à maior. Ao final da recodificação das opções de resposta, as variáveis com mais de uma opção de resposta diferente de zero foram divididas pelo número de categorias diferentes de zero, de forma que cada um do total de 33 itens variasse de 0 a 1.

Para avaliar os indicadores em cada dimensão, as respostas que apontavam a existência de capital social foram somadas, gerando uma variável quantitativa discreta. Em seguida foram calculadas as medianas e definidos os quartis para classificar os níveis de capital social. A partir dessa análise, a amostra de adolescentes foi classificada em três grupos: 1) capital social baixo (CS baixo), 25\% dos menores valores; 2) capital social moderado (CS moderado), de $25 \%$ a $75 \%$ dos valores intermediários; e capital social alto (CS alto), para valores acima de 75\% $\%^{6,24}$. A classificação do capital social total (CST) seguiu o mesmo método (Figura 2).

A distribuição dos indicadores de capital social segundo quartis está demonstrada na Figura 


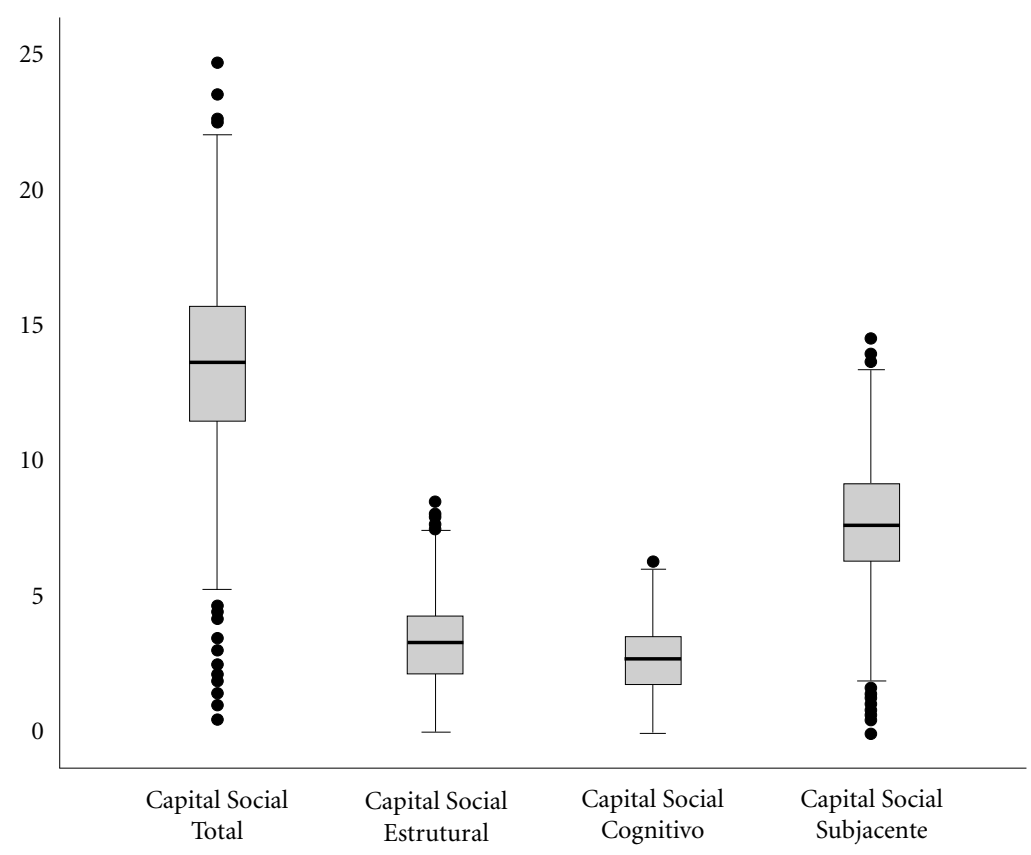

Figura 2. Distribuição das variáveis de capital social segundo quartis. RMGV-ES, 2016-2017.

Fonte: Elaborado pelos autores.

2. $\mathrm{O}$ indicador de CST apresentou percentil ${ }_{25}=$ 11,42 , mediana $=13,58$ e percentil ${ }_{75}=15,67$. O indicador de CSE revelou percentil ${ }_{25}=2,20$, mediana $=3,30$ e percentil ${ }_{75}=4,30$. Já o indicador de CSC: percentil ${ }_{25}=1,75$, mediana $=2,75$ e Percentil $_{75}=3,50$. E o CSS: percentil ${ }_{25}=6,20$, mediana $=7,60$ e percentil ${ }_{75}=9,15$. Em relação aos pontos discrepantes, para o CST e o CSS não houve pontos altamente discrepantes abaixo. CSE e CSC também não apresentaram pontos discrepantes abaixo. Em relação aos pontos discrepantes acima, para CST o ponto mais discrepante foi 24,57 . Não houve pontos altamente discrepantes acima para CSE e CSS. O CSC apresentou apenas um ponto discrepante acima.

$\mathrm{O}$ instrumento de capital social foi testado previamente por meio do método de teste e reteste, em duas etapas com intervalo de 21 dias entre as aplicações. Inicialmente, foi aplicado a 46 adolescentes de 15 e 19 anos (não incluídos no estudo principal), e então reaplicado aos mesmos estudantes, a fim de verificar no reteste inconsistências quanto às respostas dos adolescentes. Ao analisar as respostas obtidas nas duas ocasiões por meio dos testes de Kappa ${ }^{26}$ ajustado pela prevalência, os resultados demonstraram que, entre o total de 33 variáveis de capital social analisadas, oito apresentaram concordância quase perfeita (coeficiente Kappa: 0,80 - 1,0), 15 tiveram concordância substancial (coeficiente Kappa: 0,60 - 0,79), seis delas concordância moderada (coeficiente Kappa: 0,40-0,59) e três concordância fraca (coeficiente Kappa: 0,20 - 0,39). Apenas uma variável apresentou concordância muito fraca (coeficiente Kappa: 0-0,19).

Também aplicou-se o teste de McNemar às respostas, a fim de avaliar a existência de tendência de discordância nas aplicações. Verificou-se que não houve discordância estatisticamente significante, exceto na variável "De quantos grupos você, ou alguém em sua casa, faz parte?”, que demonstrou tendência de discordância significativa $(\mathrm{p}=0,025)$. Embora a tendência de discordância tenha sido significativa, a variável foi mantida conforme o instrumento original, em razão da significativa $(\mathrm{p}<0,001)$ e substancial concordância encontrada para o teste de Kappa ajustado pela prevalência $(0,76)$.

\section{Bullying}

Foi utilizada uma versão brasileira modificada do "Olweus bully/victim questionnaire", adaptada a partir de Fischer et al. ${ }^{27}$. Esses autores sistematizaram uma versão do referido instru- 
mento para a língua portuguesa a partir de dois outros trabalhos científicos ${ }^{28,29}$. O instrumento avalia a frequência, durante os últimos 12 meses, de atitudes que caracterizam comportamentos de bullying e permite a categorização dos sujeitos em vítimas e agressores ${ }^{27}$. É composto por 48 questões, sendo 24 para investigar a frequência de vitimização e 24 para a frequência de agressão, com respostas em escala tipo Likert, com seis opções de resposta $(0=$ nunca aconteceu, 1 $=$ aconteceu uma ou duas vezes no último ano, 2 $=$ aconteceu de três a seis vezes no último ano, 3 $=$ aconteceu uma vez por semana, $4=$ aconteceu várias vezes por semana e $5=$ aconteceu todos os dias). A ocorrência dos comportamentos foi categorizada em: "não ocorreu nos últimos 12 meses", "maus-tratos" (ocorreu algumas vezes nos últimos 12 meses) e "bullying" (ocorreu mais de uma vez por semana).

Foi proposta uma classificação alternativa a fim de captar a percepção dos estudantes de forma mais detalhada. Adotou-se o termo "maus-tratos" ${ }^{27}$ para se referir aos atos violentos ocorridos entre os estudantes com frequência de "algumas vezes nos últimos 12 meses", estabelecendo um critério de repetição e cronicidade igual ou menor do que seis vezes nos últimos 12 meses". O termo bullying foi adotado para se referir aos comportamentos agressivos entre estudantes que tenham ocorrido com frequência ao menos semanal, indo até uma frequência diária durante os últimos 12 meses $^{27}$.

Os parâmetros de confiabilidade ${ }^{30}$ do instrumento adaptado para identificar comportamentos de bullying foram analisados e revelaram, para os itens da vitimização, pontuação média de $0,42 \pm 0,537 \mathrm{dp}$, e para a agressão, média de 0,26 $\pm 0,432 \mathrm{dp}$. As correlações entre cada item e o valor global da escala oscilaram entre 0,284 e 0,689 para a escala de vitimização, e entre 0,320 e 0,720 para a de agressão. As escalas indicaram elevados índices de concordância (alfa de Cronbach = 0,887 para a vitimização, e alfa de Cronbach $=$ 0,888 para a agressão). Mais detalhes sobre a testagem do instrumento para medir bullying estão descritos em Reisen et al. ${ }^{31}$

\section{Análise estatística}

Análises descritivas foram realizadas para caracterizar os participantes, incluindo as frequências absolutas e relativas das variáveis analisadas e medidas de tendência central. As diferenças proporcionais entre variáveis sociodemográficas e capital social foram avaliadas pelo teste qui-qua- drado, assim como a associação entre indicadores de capital social e comportamentos de bullying. Estimativas de odds ratio (OR) e técnicas de regressão logística multinomial foram aplicadas a fim de estimar a associação entre envolvimento em comportamentos de bullying e indicadores de capital social (foi utilizado o método enter para inclusão das variáveis que apresentaram distribuição diferencial menor do que $10 \%(\mathrm{p}<0,10)$ no modelo hierárquico e considerado o intervalo de confiança de 95\%). Utilizou-se o programa Statistical Package for the Social Sciences (SPSS), versão 21.0, para a análise estatística dos dados coletados.

\section{Aspectos éticos}

O estudo foi aprovado pelo Comitê de Ética em Pesquisa do Centro de Ciências da Saúde da Universidade Federal do Espírito Santo. Todos os participantes assinaram o Termo de Consentimento Livre Esclarecido e/ou Termo de Assentimento.

\section{Resultados}

Do total de 2.293 estudantes que integraram a amostra, mais da metade tinha entre 15 e 16 anos (média de idade $=16,42 \pm 1,14 \mathrm{dp}$ ), a maioria era do sexo feminino $(\mathrm{n}=1.374 ; 60,0 \%)$ e a raçacor autodeclarada predominante foi a parda $(\mathrm{n}=$ $1.039 ; 45,4 \%)$. Entre os estudantes, $88,0 \%(\mathrm{n}=$ 2.013) eram da rede pública de ensino, mais de $80 \%(\mathrm{n}=1.867)$ estavam matriculados no turno matutino e quase metade cursava o primeiro ano do ensino médio. A maioria não possuía qualquer trabalho remunerado $(\mathrm{n}=1.758 ; 80,0 \%)$, pouco mais da metade residia no mesmo endereço há mais de dez anos, a maior parte deles relatou existir saneamento básico em suas moradias e residir com até cinco pessoas no domicílio $(\mathrm{n}=$ $1.972 ; 86,2 \%$ ). Pouco mais da metade era filho de pais divorciados $(\mathrm{n}=1.323 ; 58,5 \%)$. Quase metade relatou escolaridade fundamental do chefe da família $(\mathrm{n}=1.052 ; 47,0 \%)$ e renda familiar total de até três salários mínimos $(\mathrm{n}=793 ; 44,6 \%)$ (Tabela 1).

Ao executar as análises de associação, verificou-se que as variáveis sexo $(\mathrm{p}=0,027)$ e renda familiar total $(\mathrm{p}=0,005)$ foram significativamente associadas ao CST (Tabela 1). Contudo, ao aplicar a técnica da regressão logística multinomial às variáveis que apresentaram associação estatística significativa com o CST, apenas a vari- 
Tabela 1. Distribuição do capital social total segundo características gerais dos adolescentes. RMGV-ES, 20162017.

\begin{tabular}{|c|c|c|c|c|c|c|c|c|c|c|}
\hline & \multicolumn{9}{|c|}{ Capital Social Total } & \multirow{3}{*}{$\begin{array}{c}\text { P- } \\
\text { valor }\end{array}$} \\
\hline & \multicolumn{2}{|c|}{ Baixo } & \multirow[b]{2}{*}{ IC 95\% } & \multicolumn{2}{|c|}{ Moderado } & \multicolumn{3}{|c|}{ Alto } & \multirow[b]{2}{*}{ IC 95\% } & \\
\hline & $\mathbf{N}$ & $\%$ & & $\mathbf{N}$ & $\%$ & IC 95\% & $\mathbf{N}$ & $\%$ & & \\
\hline Idade $(\mathrm{n}=2.290)^{¥}$ & 574 & 100,0 & & 1.143 & 100,0 & & 573 & 100,0 & & 0,457 \\
\hline $\begin{array}{l}15 \text { e } 16 \text { anos } \\
(\mathrm{n}=1.271)\end{array}$ & 310 & 54,0 & $47,4-60,6$ & 656 & 57,4 & $50,8-64,0$ & 305 & 53,2 & $46,6-59,8$ & \\
\hline $17 \operatorname{anos}(\mathrm{n}=581)$ & 154 & 26,8 & $20,9-32,7$ & 276 & 24,1 & $18,4-29,8$ & 151 & 26,4 & $20,5-32,3$ & \\
\hline 18 e 19 anos $(n=438)$ & 110 & 19,2 & $14,0-24,4$ & 211 & 18,5 & $13,3-23,7$ & 117 & 20,4 & $15,0-25,8$ & \\
\hline $\operatorname{Sexo}(\mathrm{n}=2.290)^{¥}$ & 574 & 100,0 & & 1.143 & 100,0 & & 573 & 100,0 & & 0,027 \\
\hline Feminino $(\mathrm{n}=1.374)$ & 358 & 62,4 & $56,0-68,8$ & 699 & 61,2 & $54,7-67,7$ & 317 & 55,3 & $48,7-61,9$ & \\
\hline Masculino $(\mathrm{n}=916)$ & 216 & 37,6 & $31,2-44,0$ & 444 & 38,8 & $32,3-45,3$ & 256 & 44,7 & $38,1-51,3$ & \\
\hline Raça-cor $\left(\mathrm{n}=2.287^{¥}\right.$ & 573 & 100,0 & & 1.141 & 100,0 & & 573 & 100,0 & & 0,661 \\
\hline Branca $(\mathrm{n}=654)$ & 168 & 29,3 & $23,2-35,4$ & 324 & 28,4 & $22,4-34,4$ & 162 & 28,3 & $22,3-34,3$ & \\
\hline Preta $(n=405)$ & 110 & 19,2 & $14,0-24,4$ & 189 & 16,6 & $11,6-21,6$ & 106 & 18,5 & $13,3-23,7$ & \\
\hline Parda $(\mathrm{n}=1.039)$ & 242 & 42,2 & $35,6-48,8$ & 539 & 47,2 & $40,6-53,8$ & 258 & 45,0 & $38,4-51,6$ & \\
\hline Amarela $(\mathrm{n}=146)$ & 42 & 7,3 & $3,8-10,8$ & 70 & 6,1 & $2,9-9,3$ & 34 & 5,9 & $2,8-9,0$ & \\
\hline Indígena $(n=43)$ & 11 & 2,0 & $0,1-3,9$ & 19 & 1,7 & $0,0-3,4$ & 13 & 2,3 & $0,3-4,3$ & \\
\hline $\begin{array}{l}\text { Ano do ensino médio ( } \mathrm{n} \\
=2.290)^{¥}\end{array}$ & 574 & 100,0 & & 1.143 & 100,0 & & 573 & 100,0 & & 0,762 \\
\hline $\begin{array}{l}\text { Primeiro ano } \\
(\mathrm{n}=1.092)\end{array}$ & 274 & 47,7 & $41,1-54,3$ & 553 & 48,4 & $41,8-55,0$ & 265 & 46,2 & $39,6-52,8$ & \\
\hline Segundo ano $(n=619)$ & 162 & 28,2 & $22,2-34,2$ & 304 & 26,6 & $20,7-32,5$ & 153 & 26,7 & $20,8-32,6$ & \\
\hline $\begin{array}{l}\text { Terceiro e quarto } \\
\text { anos }(\mathrm{n}=579)\end{array}$ & 138 & 24,0 & $18,3-29,7$ & 286 & 25,0 & $19,2-30,8$ & 155 & 27,1 & $21,2-33,0$ & \\
\hline Turno $(\mathrm{n}=2.277)^{¥}$ & 573 & 100,0 & & 1.136 & 100,0 & & 568 & 100,0 & & 0,960 \\
\hline Manhã $(\mathrm{n}=1.867)$ & 469 & 81,8 & $76,7-86,9$ & 934 & 82,2 & $77,1-87,3$ & 464 & 81,7 & $76,6-86,8$ & \\
\hline Tarde $(\mathrm{n}=410)$ & 104 & 18,2 & $13,1-23,3$ & 202 & 17,8 & $12,7-22,9$ & 104 & 18,3 & $13,2-23,4$ & \\
\hline $\begin{array}{l}\text { Rede de ensino } \\
(\mathrm{n}=2.289)^{¥}\end{array}$ & 574 & 100,0 & & 1.142 & 100,0 & & 573 & 100,0 & & 0,440 \\
\hline Pública $(\mathrm{n}=2.013)$ & 512 & 89,2 & $85,1-93,3$ & 1004 & 87,9 & $83,6-92,2$ & 497 & 86,6 & $82,1-91,1$ & \\
\hline Privada $(\mathrm{n}=276)$ & 62 & 10,8 & $6,7-14,9$ & 138 & 12,1 & $7,8-16,4$ & 76 & 13,4 & $8,9-17,9$ & \\
\hline $\begin{array}{l}\text { Trabalho remunerado do } \\
\text { adolescente }(\mathrm{n}=2.289)^{¥}\end{array}$ & 573 & 100,0 & & 1.143 & 100,0 & & 573 & 100,0 & & 0,444 \\
\hline Não $(\mathrm{n}=1.758)$ & 445 & 77,7 & $72,2-83,2$ & 884 & 77,3 & $71,7-82,9$ & 429 & 74,9 & $69,1-80,7$ & \\
\hline $\operatorname{Sim}(n=531)$ & 128 & 22,3 & $16,8-27,8$ & 259 & 22,7 & $17,1-28,3$ & 144 & 25,1 & $19,3-30,9$ & \\
\hline $\begin{array}{l}\text { Anos de residência no } \\
\text { endereço }(\mathrm{n}=2.267)^{¥}\end{array}$ & 567 & 100,0 & & 1.134 & 100,0 & & 566 & 100,0 & & 0,280 \\
\hline Até 10 anos $(\mathrm{n}=1.107)$ & 293 & 51,7 & $45,1-58,3$ & 540 & 47,6 & $41,0-54,2$ & 274 & 48,4 & $41,8-55,0$ & \\
\hline $\begin{array}{l}\text { Acima de } 10 \text { anos } \\
(\mathrm{n}=1.160)\end{array}$ & 274 & 48,3 & $41,7-54,9$ & 594 & 52,4 & $45,8-59,0$ & 292 & 51,6 & $45,0-58,2$ & \\
\hline
\end{tabular}

ável sexo permaneceu associada $(\mathrm{p}=0,021)$, com OR $=1,4$ (IC 95\% = 1,05-1,80), revelando chance $40,0 \%$ maior das estudantes do sexo feminino apresentarem CS baixo, quando comparado aos estudantes do sexo masculino com CS alto (resultado não apresentado em tabela).

A Tabela 2 demostra os resultados da associação entre os indicadores de capital social e comportamentos de bullying entre os adolescentes.
Ao observar a relação dos indicadores de CSC ( $\mathrm{p}$ $=0,002)$ e CSS $(\mathrm{p}=0,007)$ com a vitimização, nota-se que, à medida em que o nível de capital social se elevou, a frequência de bullying diminuiu. Em relação ao CST ( $\mathrm{p}<0,001)$, verifica-se que a relação com a vitimização de bullying indica percentuais semelhantes aos níveis de CS baixo e alto. No que se refere às associações com comportamentos agressivos, foram estatisticamente 
Tabela 1. Distribuição do capital social total segundo características gerais dos adolescentes. RMGV-ES, 20162017.

\begin{tabular}{|c|c|c|c|c|c|c|c|c|c|c|}
\hline & \multicolumn{9}{|c|}{ Capital Social Total } & \multirow{3}{*}{$\begin{array}{c}\text { P- } \\
\text { valor }^{*}\end{array}$} \\
\hline & \multicolumn{2}{|c|}{ Baixo } & \multirow[b]{2}{*}{ IC 95\% } & \multicolumn{2}{|c|}{ Moderado } & \multicolumn{3}{|c|}{ Alto } & \multirow[b]{2}{*}{ IC 95\% } & \\
\hline & $\mathbf{N}$ & $\%$ & & $\mathbf{N}$ & $\%$ & IC $95 \%$ & $\mathbf{N}$ & $\%$ & & \\
\hline $\begin{array}{l}\text { Saneamento básico } \\
(\mathrm{n}=2.207)^{¥}\end{array}$ & 537 & 100,0 & & 1.113 & 100,0 & & 557 & 100,0 & & 0,848 \\
\hline Não $(\mathrm{n}=528)$ & 126 & 23,5 & $17,9-29,1$ & 272 & 24,4 & $18,7-30,1$ & 130 & 23,3 & $17,7-28,9$ & \\
\hline $\operatorname{Sim}(n=1.679)$ & 411 & 76,5 & $70,9-82,1$ & 841 & 75,6 & $69,9-81,3$ & 427 & 76,4 & $70,8-82,0$ & \\
\hline $\begin{array}{l}\text { Número de moradores no } \\
\text { domicílio }(\mathrm{n}=2.289)^{¥}\end{array}$ & 574 & 100,0 & & 1.143 & 100,0 & & 572 & 100,0 & & 0,869 \\
\hline $\begin{array}{l}\text { Até } 5 \text { pessoas } \\
(\mathrm{n}=1.972)\end{array}$ & 496 & 86,4 & $81,8-91,0$ & 987 & 86,4 & $81,8-91,0$ & 489 & 85,5 & $80,8-90,2$ & \\
\hline $\begin{array}{l}\text { Mais de } 5 \text { pessoas } \\
(\mathrm{n}=317)\end{array}$ & 78 & 13,6 & $9,0-18,2$ & 156 & 13,6 & $9,0-18,2$ & 83 & 14,5 & $9,8-19,2$ & \\
\hline $\begin{array}{l}\text { Divórcio parental } \\
(\mathrm{n}=2.263)^{¥}\end{array}$ & 559 & 100,0 & & 1.137 & 100,0 & & 567 & 100,0 & & 0,153 \\
\hline Não $(\mathrm{n}=1.323)$ & 327 & 58,5 & $51,9-65,1$ & 646 & 56,8 & $50,2-63,4$ & 350 & 61,7 & $55,2-68,2$ & \\
\hline $\operatorname{Sim}(n=940)$ & 232 & 41,5 & $34,9-48,1$ & 491 & 43,2 & $36,6-49,8$ & 217 & 38,3 & $31,8-44,8$ & \\
\hline $\begin{array}{l}\text { Escolaridade do chefe da } \\
\text { família }(\mathrm{n}=2.242)^{¥}\end{array}$ & 553 & 100,0 & & 1.125 & 100,0 & & 564 & 100,0 & & 0,315 \\
\hline $\begin{array}{l}\text { Fundamental } \\
(\mathrm{n}=1.052)\end{array}$ & 262 & 47,4 & $40,8-54,0$ & 525 & 46,7 & $40,1-53,5$ & 265 & 47,0 & $40,4-53,6$ & \\
\hline Médio $(\mathrm{n}=766)$ & 193 & 34,9 & $28,6-41,2$ & 396 & 35,2 & $28,8-41,6$ & 177 & 31,4 & $25,2-37,6$ & \\
\hline Superior $(\mathrm{n}=424)$ & 98 & 17,7 & $12,6-22,8$ & 204 & 18,1 & $13,0-23,2$ & 122 & 21,6 & $16,1-27,1$ & \\
\hline $\begin{array}{l}\text { Renda familiar total } \\
(\mathrm{n}=1.778)^{¥ \S}\end{array}$ & 423 & 100,0 & & 899 & 100,0 & & 456 & 100,0 & & 0,005 \\
\hline $\begin{array}{l}\text { Até } 1 \text { salário mínimo } \\
(\mathrm{n}=225)\end{array}$ & 60 & 14,2 & $12,6-22,8$ & 104 & 11,6 & $7,3-15,9$ & 61 & 13,4 & $8,9-17,9$ & \\
\hline $\begin{array}{l}\text { Mais de } 1 \text { até } 3 \text { salários } \\
\text { mínimos }(\mathrm{n}=793)\end{array}$ & 180 & 42,6 & $9,6-18,8$ & 442 & 49,2 & $42,5-55,9$ & 171 & 37,5 & $31,1-43,9$ & \\
\hline $\begin{array}{l}\text { Mais de } 3 \text { até } 10 \text { salários } \\
\text { mínimos }(\mathrm{n}=642)\end{array}$ & 154 & 36,4 & $30,0-42,8$ & 298 & 33,1 & $26,8-39,4$ & 190 & 41,7 & $35,1-48,3$ & \\
\hline $\begin{array}{l}\text { Mais de } 10 \text { salários } \\
\text { mínimos }(\mathrm{n}=118)\end{array}$ & 29 & 6,8 & $3,5-10,1$ & 55 & 6,1 & $2,9-9,3$ & 34 & 7,4 & $3,9-10,9$ & \\
\hline
\end{tabular}

significantes CSC $(\mathrm{p}<0,001)$ e CST $(\mathrm{p}=0,011)$. Atos de perpetração por bullying demonstraram redução percentual à medida que o capital social se eleva.

A Tabela 3 apresenta o modelo final ajustado de regressão logística multinomial entre as variáveis bullying (dependente) e capital social (variável independente). Ao analisar os resultados das razões de chance encontradas para vitimização, verificou-se que houve associação estatística significante em todos os indicadores testados. O indicador de CSC demostrou que os adolescentes que possuem CS baixo [p $=0,001, \mathrm{OR}=1,9$ (IC $95 \%=1,29-2,68)]$ e moderado $[\mathrm{p}=0,005, \mathrm{OR}=$ $1,5($ IC $95 \%=1,13-1,99)]$ têm mais chances de ser vítimas de bullying do que aqueles que possuem CS alto. Situação também identificada no CSS e no CST.

Entre os comportamentos agressivos de bullying também foi possível observar associação estatística significante com o capital social em todos os indicadores analisados (Tabela 3). Os adolescentes que possuíam CSC baixo $[\mathrm{p}<0,001$, $\mathrm{OR}=3,2(\mathrm{IC} 95 \%=2,34-4,44)$ e moderado $[\mathrm{p}<$ $0,001, \mathrm{OR}=1,8($ IC $95 \%=1,38-2,34)]$ tiveram maiores chances de ser agressores de bullying do que aqueles que possuíam CS alto. Situação que se repetiu com o CST baixo [ $\mathrm{p}=0,001, \mathrm{OR}=1,7$ (IC 95\% = 1,24-2,27)] e moderado $[\mathrm{p}=0,042$, $\mathrm{OR}=1,3(\mathrm{IC} 95 \%=1,01-1,72)]$. 
Tabela 2. Associação entre capital social e comportamentos de bullying. RMGV-ES, 2016-2017.

\begin{tabular}{|c|c|c|c|c|c|c|c|}
\hline & \multicolumn{7}{|c|}{ Vitimização } \\
\hline & \multicolumn{2}{|c|}{ Nunca aconteceu } & \multicolumn{2}{|c|}{ Maus-tratos } & \multicolumn{2}{|c|}{ Bullying } & \multirow{2}{*}{ P-valor $\times$} \\
\hline & $\mathbf{N}$ & $\%$ & $\mathbf{N}$ & $\%$ & $\mathbf{N}$ & $\%$ & \\
\hline Total $(\mathrm{n}=2.282)$ & 356 & 15,6 & 989 & 43,3 & 937 & 41,1 & \\
\hline Indicador de capital social estrutural & & & & & & & 0,767 \\
\hline Baixo $(n=570)$ & 88 & 15,4 & 244 & 42,8 & 238 & 41,8 & \\
\hline Moderado $(\mathrm{n}=1.152)$ & 184 & 16,0 & 510 & 42,3 & 458 & 39,7 & \\
\hline Alto $(\mathrm{n}=560)$ & 84 & 15,0 & 235 & 42,0 & 241 & 43,0 & \\
\hline Indicador de capital social cognitivo & & & & & & & 0,002 \\
\hline Baixo $(n=454)$ & 61 & 13,4 & 180 & 39,7 & 213 & 46,9 & \\
\hline Moderado $(\mathrm{n}=1.229)$ & 182 & 14,8 & 535 & 43,5 & 512 & 41,7 & \\
\hline Alto $(\mathrm{n}=599)$ & 113 & 18,9 & 274 & 45,7 & 212 & 35,4 & \\
\hline Indicador de capital social subjacente & & & & & & & 0,007 \\
\hline Baixo $(\mathrm{n}=561)$ & 80 & 14,3 & 226 & 40,3 & 255 & 45,4 & \\
\hline Moderado $(\mathrm{n}=1.146)$ & 165 & 14,4 & 508 & 44,3 & 473 & 41,3 & \\
\hline Alto $(\mathrm{n}=575)$ & 111 & 19,3 & 255 & 44,3 & 209 & 36,4 & \\
\hline Capital social total & & & & & & & $<0,001$ \\
\hline Baixo $(n=567)$ & 80 & 14,1 & 227 & 40,0 & 260 & 45,9 & \\
\hline Moderado $(\mathrm{n}=1.143)$ & 159 & 13,9 & 513 & 44,9 & 471 & 41,2 & \\
\hline \multirow[t]{4}{*}{ Alto $(\mathrm{n}=572)$} & 117 & 20,5 & 249 & 43,5 & 206 & 45,9 & \\
\hline & \multicolumn{7}{|c|}{ Agressão } \\
\hline & \multicolumn{2}{|c|}{ Nunca aconteceu } & \multicolumn{2}{|c|}{ Maus-tratos } & \multicolumn{2}{|c|}{ Bullying } & D nolor \\
\hline & $\mathbf{N}$ & $\%$ & $\mathbf{N}$ & $\%$ & $\mathbf{N}$ & $\%$ & P-valor \\
\hline Total $(\mathrm{n}=2.281)$ & 699 & 30,7 & 920 & 40,3 & 662 & 29,0 & \\
\hline Indicador de capital social estrutural & & & & & & & 0,065 \\
\hline Baixo $(\mathrm{n}=570)$ & 195 & 34,2 & 218 & 38,2 & 157 & 27,5 & \\
\hline Moderado $(\mathrm{n}=1.151)$ & 357 & 31,0 & 466 & 40,5 & 328 & 28,5 & \\
\hline Alto $(\mathrm{n}=560)$ & 147 & 26,3 & 236 & 42,1 & 177 & 31,6 & \\
\hline Indicador de capital social cognitivo & & & & & & & $<0,001$ \\
\hline Baixo $(\mathrm{n}=454)$ & 108 & 23,8 & 158 & 34,8 & 188 & 41,4 & \\
\hline Moderado $(\mathrm{n}=1.228)$ & 358 & 29,2 & 522 & 42,5 & 348 & 28,3 & \\
\hline Alto $(\mathrm{n}=599)$ & 233 & 38,9 & 240 & 40,1 & 126 & 21,0 & \\
\hline Indicador de capital social subjacente & & & & & & & 0,326 \\
\hline Baixo $(\mathrm{n}=561)$ & 157 & 28,0 & 233 & 41,5 & 171 & 30,5 & \\
\hline Moderado $(\mathrm{n}=1.145)$ & 350 & 30,6 & 457 & 39,9 & 338 & 29,5 & \\
\hline Alto $(\mathrm{n}=575)$ & 192 & 33,4 & 230 & 40,0 & 153 & 26,6 & \\
\hline Capital social total & & & & & & & 0,011 \\
\hline Baixo $(\mathrm{n}=566)$ & 158 & 27,9 & 217 & 38,3 & 191 & 33,8 & \\
\hline Moderado $(\mathrm{n}=1.143)$ & 350 & 30,6 & 460 & 40,3 & 333 & 29,1 & \\
\hline Alto $(\mathrm{n}=572)$ & 191 & 33,4 & 243 & 42,5 & 138 & 24,1 & \\
\hline
\end{tabular}

* Teste qui-quadrado de Pearson.

Fonte: Elaborado pelos autores.

Entretanto, os estudantes que possuíam CSE baixo $[\mathrm{p}=0,009$, OR $=0,7($ IC 95\% $=0,49-0,91)$ ] e moderado $[\mathrm{p}=0,046, \mathrm{OR}=0,8$ (IC $95 \%=$ $0,58-0,99)]$ tiveram menores chances de ser perpetradores de bullying quando comparados aos que possuíam CSE alto.

\section{Discussão}

Os resultados indicaram que menores níveis de capital social estão associados a maiores chances de os adolescentes serem vítimas e agressores de bullying. Por meio deste estudo, foram abordados aspectos pouco investigados em um país em desenvolvimento, como o Brasil, marcado por am- 
Tabela 3. Associações entre comportamentos de bullying com capital social. RMGV-ES, 2016-2017.

\begin{tabular}{|c|c|c|c|}
\hline & \multicolumn{3}{|c|}{ Vitimização } \\
\hline & & Maus-tratos & Bullying \\
\hline \multirow{8}{*}{$\begin{array}{l}\text { Indicador de capital } \\
\text { social cognitivo }\end{array}$} & Baixo & 180 & 213 \\
\hline & & $\mathrm{OR}=1,2(\mathrm{IC} 95 \%=0,85-1,75)$ & $\mathrm{OR}=1,9(\mathrm{IC} 95 \%=1,29-2,68)$ \\
\hline & & $P=0,290$ & $\mathrm{P}=0,001$ \\
\hline & Moderado & 535 & 512 \\
\hline & & $\mathrm{OR}=1,2(\mathrm{IC} 95 \%=0,92-1,6)$ & $\mathrm{OR}=1,5(\mathrm{IC} 95 \%=1,13-1,99)$ \\
\hline & & $\mathrm{p}=0,172$ & $\mathrm{p}=0,005$ \\
\hline & Alto & 274 & 212 \\
\hline & & $\mathrm{OR}=1$ & $\mathrm{OR}=1$ \\
\hline \multirow{8}{*}{$\begin{array}{l}\text { Indicador de capital } \\
\text { social subjacente }\end{array}$} & Baixo & 226 & 255 \\
\hline & & $\mathrm{OR}=1,2(\mathrm{IC} 95 \%=0,88-1,73)$ & $\mathrm{OR}=1,7(\mathrm{IC} 95 \%=1,20-2,38)$ \\
\hline & & $\mathrm{p}=0,231$ & $\mathrm{p}=0,002$ \\
\hline & Moderado & 508 & 473 \\
\hline & & $\mathrm{OR}=1,3(\mathrm{IC} 95 \%=1,01-1,78)$ & $\mathrm{OR}=1,5(\mathrm{IC} 95 \%=1,14-2,04)$ \\
\hline & & $\mathrm{P}=0,043$ & $\mathrm{p}=0,005$ \\
\hline & Alto & 255 & 209 \\
\hline & & $\mathrm{OR}=1$ & $\mathrm{OR}=1$ \\
\hline \multirow[t]{10}{*}{ Capital social total } & Baixo & 227 & 260 \\
\hline & & $\mathrm{OR}=1,3(\mathrm{IC} 95 \%=0,95-1,87)$ & $\mathrm{OR}=1,8(\mathrm{IC} 95 \%=1,32-2,59)$ \\
\hline & & $\mathrm{p}=0,094$ & $\mathrm{p}<0,0001$ \\
\hline & Moderado & 513 & 471 \\
\hline & & $\mathrm{OR}=1,5(\mathrm{IC} 95 \%=1,14-2,01)$ & $\mathrm{OR}=1,7(\mathrm{IC} 95 \%=1,26-2,25)$ \\
\hline & & $\mathrm{p}=0,004$ & $\mathrm{p}<0,0001$ \\
\hline & Alto & 249 & 206 \\
\hline & & $\mathrm{OR}=1$ & $\mathrm{OR}=1$ \\
\hline & & Agressão & \\
\hline & & Maus-tratos & Bullying \\
\hline \multirow{8}{*}{$\begin{array}{l}\text { Indicador de capital } \\
\text { social estrutural }\end{array}$} & Baixo & 218 & 157 \\
\hline & & $\mathrm{OR}=0,7(\mathrm{IC} 95 \%=0,53-0,92)$ & $\mathrm{OR}=0,7(\mathrm{IC} 95 \%=0,49-0,91)$ \\
\hline & & $\mathrm{p}=0,012$ & $\mathrm{p}=0,009$ \\
\hline & Moderado & 466 & 328 \\
\hline & & $\mathrm{OR}=0,8(\mathrm{IC} 95 \%=0,64-1,04)$ & $\mathrm{OR}=0,8(\mathrm{IC} 95 \%=0,58-0,99)$ \\
\hline & & $\mathrm{p}=0,102$ & $\mathrm{p}=0,046$ \\
\hline & Alto & 236 & 177 \\
\hline & & $\mathrm{OR}=1$ & $\mathrm{OR}=1$ \\
\hline \multirow{8}{*}{$\begin{array}{l}\text { Indicador de capital } \\
\text { social cognitivo }\end{array}$} & Baixo & 158 & 188 \\
\hline & & $\mathrm{OR}=1,4(\mathrm{IC} 95 \%=1,05-1,92)$ & $\mathrm{OR}=3,2(\mathrm{IC} 95 \%=2,34-4,44)$ \\
\hline & & $\mathrm{p}=0,024$ & $\mathrm{p}<0,001$ \\
\hline & Moderado & 522 & 348 \\
\hline & & $\mathrm{OR}=1,4(\mathrm{IC} 95 \%=1,13-1,77)$ & $\mathrm{OR}=1,8(\mathrm{IC} 95 \%=1,38-2,34)$ \\
\hline & & $\mathrm{p}=0,002$ & $\mathrm{p}<0,001$ \\
\hline & Alto & 240 & 126 \\
\hline & & $\mathrm{OR}=1$ & $\mathrm{OR}=1$ \\
\hline \multirow[t]{8}{*}{ Capital social total } & Baixo & 217 & 191 \\
\hline & & $\mathrm{OR}=1,1(\mathrm{IC} 95 \%=0,82-1,43)$ & $\mathrm{OR}=1,7(\mathrm{IC} 95 \%=1,24-2,27)$ \\
\hline & & $\mathrm{p}=0,591$ & $\mathrm{p}=0,001$ \\
\hline & Moderado & 460 & 333 \\
\hline & & $\mathrm{OR}=1,0(\mathrm{IC} 95 \%=0,82-1,31)$ & $\mathrm{OR}=1,3(\mathrm{IC} 95 \%=1,01-1,72)$ \\
\hline & & $\mathrm{p}=0,786$ & $\mathrm{p}=0,042$ \\
\hline & Alto & 243 & 138 \\
\hline & & $\mathrm{OR}=1$ & $\mathrm{OR}=1$ \\
\hline
\end{tabular}

* Categoria de referência: “Nunca aconteceu”. Resultados obtidos a partir de regressão logística multinomial (nível de significância estatística menor que $5 \%$ ).

Fonte: Elaborado pelos autores. 
plas disparidades socioeconômicas ${ }^{32}$. Também foram investigadas as associações com o bullying, comportamento potencialmente prejudicial à saúde física e mental de adolecentes, a fim de analisar a influência do capital social na determinação de comportamentos de bullying.

Entre as características gerais analisadas, apenas a variável sexo se manteve associada ao modelo final, com maior chance do sexo feminino apresentar CST baixo. Tal achado pode estar historicamente relacionado à desigualdade de gêne$\mathrm{ro}^{33}$ do sexo feminino em relação ao masculino na sociedade brasileira, seja quanto a condições mentais e físicas de saúde, acesso a serviços ou a questões associadas à ocupação no mercado de trabalho e aos rendimentos, entre outras, indicando que as desvantagens do sexo feminino podem se refletir também no nível de capital social $^{25,34,35}$.

Considerando que o capital social é um bem coletivo e que o CSC está relacionado a aspectos e comportamentos que envolvem confiança interpessoal e solidariedade, e que o CSS abrange a disponibilidade do capital social e suas formas de manifestação ${ }^{5,6}$, uma possível explicação para os achados que indicaram redução da vitimização por bullying à medida que os referidos indicadores se elevaram, diz respeito ao fato de que coletividades com níveis de capital social mais elevados difundem mais rapidamente informações dentro da comunidade, e altos níveis de confiança estimulam a adoção de novos comportamentos ${ }^{9,21}$, inclusive com relação às experiências de vitimização por bullying.

Gottfredson e DiPietro ${ }^{8}$ mostraram que frequentar ambientes escolares em que existe maior consenso sobre o que constitui um comportamento adequado influencia os episódios de vitimização, o que por sua vez reforça a importância do capital social também dentro das escolas. Nesse sentido, níveis elevados de capital social poderiam favorecer a criação de conexões sociais fortes entre os estudantes, gerando ambientes de confiança e seguros, impedindo a vitimização por bullying ${ }^{21,22}$.

Segundo alguns autores ${ }^{2,10}$, o capital social proveniente da família tem papel importante no desenvolvimento e manutenção do capital social de crianças e adolescentes, para que suas diferentes formas se estendam a contextos sociais mais amplos de convivências, gerando benefícios para o desempenho escolar e cognitivo.

As análises associativas entre capital social e vitimização por bullying revelaram que os adolescentes com CSC baixo apresentaram chance
90,0\% maior de terem sido vítimas de bullying, enquanto os estudantes com CSS e CST baixo revelaram, respectivamente, chances $70,0 \%$ e $80,0 \%$ maiores de terem sido vítimas de bullying, quando comparados aos adolescentes com CS alto. O capital social oferece ao indivíduo o sentimento de pertencimento ao grupo, e os aspectos da estrutura social que o compõem influenciam a qualidade das relações formadas nos grupos sociais, o engajamento dos indivíduos, a colaboração mútua, a confiança e a empatia ${ }^{2,5,12,21,36}$, elementos que poderiam explicar os motivos pelos quais níveis reduzidos de capital social favorecem a vitimização por bullying, enquanto níveis elevados de capital social estariam vinculados à redução da ocorrência de vitimização.

Outro possível fator explicativo estaria relacionado à disponibilidade de recursos sociais para os adolescentes, visto que pesquisadores têm sugerido que indivíduos que relatam vitimização podem ser mais vulneráveis, devido à falta de apoio de amigos próximos e à ausência de apoio de outros estudantes no ambiente escolar ${ }^{9,20}$. Relações positivas dentro dos ambientes escolares, com colegas e professores, estariam relacionadas à maior coesão entre os estudantes nesses ambientes e à redução de comportamentos violentos ${ }^{7,22}$.

Quanto à associação com os comportamentos agressivos, o fato de eles diminuírem à medida que o CSC e o CST se elevaram em nível pode estar associado a um controle mais rigoroso sobre comportamentos agressivos desviantes em comunidades escolares com maiores níveis de capital social ${ }^{8,21}$, visto que a existência de normas claras para o comportamento colaborativo adequado direcionadas aos perpetradores de bullying potenciais dentro dos ambientes escolares são importantes para a neutralização da agressividade ${ }^{8}$.

Por outro lado, o achado que apontou menor prática de bullying entre estudantes com CSE baixo pode estar relacionado ao envolvimento reduzido dos adolescentes em práticas coletivas, características do capital social, e à limitada sensação de pertencimento grupal ${ }^{2,5,36}$, produzindo comportamentos que favorecem a redução da prática de bullying e que podem estar associados à fragilidade das relações sociais interpessoais de alguns indivíduos. Esses achados apontam para a importância dos elementos do capital social nas relações sociais e nos espaços de convivência dos adolescentes, bem como para a necessidade de outros estudos específicos sobre o tema.

Em relação aos atos de perpetração de bullying, o modelo final de regressão logísti- 
ca multinomial demonstrou que os estudantes com CSC baixo apresentaram chances três vezes maiores de terem sido agressores de bullying. Já os adolescentes identificados como CST baixo revelaram chances $70,0 \%$ maiores de envolvimento como agressores de bullying do que aqueles com CS alto. O CSC está relacionado ao grau de confiança e solidariedade interpessoais ${ }^{5}$, dessa forma, o aumento e o fortalecimento desses laços estariam vinculados à redução de comportamentos desviantes, entre os quais se incluem episódios de agressão por bullying contra outros estudantes ${ }^{7}$. Se os estudantes fortalecem os laços sociais por meio de diferentes caminhos, como a participação em atividades escolares ou com normas de grupo, a confiança no ambiente escolar aumenta, em relação a professores e outros estudantes, de forma que se presume que as agressões por bullying diminuam. McPherson e colaboradores $^{10}$, em revisão sistemática, avaliaram a influência do capital social no comportamento de crianças e adolescentes, revelando que esse fornece proteção aos adolescentes contra comportamentos agressivos tanto na escola como na comunidade.

A relação entre agressores de bullying e CSE mostrou-se uma exceção em relação às demais análises, visto que níveis baixos e moderados do referido capital social evitariam a perpetração de bullying pelos estudantes, quando comparado àqueles com CSE alto. Isso evidencia que pode haver uma formação de grupos e redes sociais entre os adolescentes, que se aglutinariam para a perpetração do bullying e utilizariam o capital social de uma forma negativa ${ }^{5}$, já que nesse caso os estudantes com alto CSE agrediram mais do que estudantes com menos CSE. Outra possibilidade explicativa pode estar associada à existência de conexões sociais satisfatórias entre os indivíduos não refletidas nos níveis de capital social avaliados, assim eles teriam desenvolvido comportamentos sociais que evitaram a prática de agressão a outros estudantes.

Algumas limitações devem ser consideradas no presente estudo. Entre as pesquisas que exploram elementos do capital social, há pouca padronização em relação aos instrumentos e critérios utilizados, o que dificulta a adequada comparação entre os resultados. $\mathrm{O}$ método aplicado pode ter sido muito sensível mas pouco específico para medir o capital social. Outra lacuna está associada à reduzida participação das escolas da rede particular de ensino, que em sua maioria se negaram a participar. Além disso, os dados foram coletados por meio de autorrelato em um único momento.
Contudo, o estudo abordou relações carentes de pesquisas científicas ao explorar as associações entre indicadores de capital social e comportamentos de bullying na adolescência tardia.

Embora o aumento do número de estudos que avaliem a influência do capital social no desenvolvimento e comportamento de adolescentes tenha avançado nos últimos anos, ainda é limita$\mathrm{do}^{7,22,25}$. Os comportamentos de bullying transcendem a área educacional e alcançam a da saúde coletiva, por isso deveriam compor a agenda dos profissionais que atuam na saúde, especialmente daqueles que lidam com crianças e adolescentes, grupo mais vulnerável às ocorrências ${ }^{13,16}$.

Enquanto sociedade, medidas educativas deveriam ser adotadas para que os adolescentes compreendam a influência que cada um deles pode ter na vida do outro e nos ambientes que convivem, por meio do estímulo ao senso de responsabilidade e a iniciativas anti-bullying, em nível individual, de grupo e/ou escolar ${ }^{9,13,15,19,21}$.

Não foram identificados estudos científicos prévios que avaliassem a influência do capital social na determinação de comportamentos de bullying no cenário brasileiro. O presente estudo aponta para o capital social como um fator influenciador na ocorrência de comportamentos agressivos entre estudantes. Tal fato favorece o embasamento para o desenvolvimento de ações e práticas interdisciplinares/intersetoriais para o enfrentamento do bullying, de forma local ou populacional, a partir da promoção de mudanças, sobretudo comportamentais, voltadas à convivência pacífica, ao protagonismo juvenil e ao bem comum, favorecendo a saúde coletiva, tendo em vista que a prevenção do bullying evita problemas de saúde física e mental (como alterações do sono, cefaleia, desmaios, vômitos, paralisias, anorexia, bulimia, consumo de álcool e drogas, agressividade, criminalidade, traumas, baixa autoestima, baixa confiança nas pessoas, ansiedade, depressão, dificuldades de socialização, pânico e maior tendência ao suicídio $)^{13,16}$ na vida adulta.

As evidências permitiram concluir que, à medida que o capital social se elevou em nível, os comportamentos de bullying diminuíram, demonstrando sua influência nos comportamentos de vitimização e de agressão entre estudantes na adolescência. Em relação aos indicadores específicos, essa associação se manteve para CSS, na vitimização, e para CSC, na vitimização e na agressão. Entretanto, a relação entre CSE e agressão por bullying destaca a necessidade de estudos futuros que a explorem de forma mais aprofundada. 


\section{Colaboradores}

A Reisen: concepção, planejamento, análise, interpretação dos resultados e redação do trabalho. FMC Leite: análise, interpretação dos resultados e revisão final do trabalho. ET Santos Neto: concepção, planejamento, interpretação dos resultados, redação e revisão final do trabalho. Todos os autores aprovaram a versão final encaminhada.

\section{Referências}

1. Celeste RK, Nadanovsky P. Aspectos relacionados aos efeitos da desigualdade de renda na saúde: mecanismos contextuais. Cien Saude Colet 2010; 15(5):25072519.

2. Coleman JS. Social Capital in the creation of human capital. Am J Sociol 1988; 94:95-120.

3. Putnam RD. Bowling alone: the collapse and revival of American community. New York: Simon and Schuster; 2000.

4. Portes A. Social Capital: Its origins and applications in contemporary sociology. Annual reviews 1998; 24:124.

5. Grootaert C, Narayan D, Jones V, Woolcook M. Measuring social capital: an integrated questionnaire. Washington: World Bank; 2003.

6. Vial EA, Junges JR, Olinto MTA, Machado PS, Pattussi MP. Violência urbana e capital social em uma cidade no Sul do Brasil: um estudo quantitativo e qualitativo. Rev Panam Salud Publica 2010; 28(4):289-297.

7. Kirk DS. Unraveling the contextual effects on student suspension and juvenile arrest: an examination of school, neighborhood, and family controls. Crimino$\log y$ 2008; 47(2):479-520.

8. Gottfredson DC, DiPietro SM. School size, social capital, and student victimization. Sociol Educ 2011; 84(1):69-89.

9. Carney JV, Jacob CJ, Hazler RJ. Exposure to school bullying and the social capital of sixth-grade students. J Humanist Couns 2011; 50(2):238-253.

10. McPherson K, Kerr S, McGee E, Cheater F, Morgan A. The role and impact of social capital on the health and wellbeing of children and adolescents: a systematic review. Glasgow: Glasgow Caledonian University; 2013.

11. Solar O, Irwin A. A conceptual framework for action on the social determinants of health. social determinants of health discussion paper 2. Geneva: World Health Organization; 2010.

12. Vieno A, Lenzi M, Roccato M, Russo S, Monaci MG, Scacchi L. Social capital and fear of crime in adolescence: a multilevel study. Am J Community Psychol 2016; 58(1-2):100-110.

13. Lopes-Neto AA. Bullying aggressive behavior among students. J Pediatr 2005; 81(5):164-172.

14. Olweus D. School bullying: development and some important challenges. Annu Rev Clin Psychol 2013; 9:751-780.

15. Arseneault L. Annual research review: the persistent and pervasive impact of being bullied in childhood and adolescence: implication for policy and practice. J. Child Psychol Psychiatry 2018; 59(4):405-421.

16. Silva ABB. Bullying: mentes perigosas nas escolas. São Paulo: Globo Livro; 2015.

17. Oliveira WA, Silva MAI, Silva JL, Mello FCM, Prado RR, Malta DC. Associations between the practice of bullying and individual and contextual variables from the aggressors' perspective. J Pediatr 2016; 92(1):3239 .

18. Mello FCM, Silva JL, Oliveira WA, Prado RR, Malta DC, Silva MAI. A prática de bullying entre escolares brasileiros e fatores associados, Pesquisa Nacional de Saúde do Escolar 2015. Cien Saude Colet 2017; 22(9):2939-2948. 
19. Mota RS, Gomes NP, Campos LM, Cordeiro KCC, Souza CNP, Camargo L. Adolescentes escolares: associação entre vivência de bullying e consumo de álcool/ drogas. Texto Contexto Enferm 2018; 27(3):1-10.

20. Demaray MK, Malecki CK. Perceptions of the frequency and importance of social support by students classified as victims, bullies, and bully/victims in an urban middle school. School Psych Rev 2003; 32(3):471-489.

21. Carney JV, Liu Y, Hazler RJ. A path analysis on school bullying and critical school environment variables: a social capital perspective. Child Youth Serv Rev 2018; 93:231-239.

22. Sobba K. Using social capital to inform policy regarding bullying victimization. Sociation Today [periódico na Internet] 2017; 15(2). [acessado 2017 Mai 16]. Disponível em: http://www.ncsociology.org/sociationtoday /v152/sobba.html.

23. Instituto Brasileiro de Geografia e Estatística (IBGE). Censo demográfico. Brasília: IBGE; 2010.

24. Reisen A, Viana MC, Santos-Neto ET. Adverse childhood experiences and bullying in late adolescence in a metropolitan region of Brazil. Child Abuse Negl 2019; 92:146-156.

25. Bezerra IA, Goes PSA. Associação entre capital social, condições e comportamentos de saúde bucal. Cienc Saude Colet 2014; 19(6):1943-1950.

26. Landis JR, Koch GG. The measurement of observer agreement for categorical data. Biometrics 1977; 33:159-75.

27. Centro de Empreendedorismo Social e Administração em Terceiro Setor (CEATS), Fundação Instituto de Administração (FIA). Bullying escolar no Brasil relatório final. [acessado 2018 Jul 9]. Disponível em: http://www.ucb.br/sites/100/127/documentos/bibliotecal.pdf

28. Fante CAZ. Fenômeno bullying: como prevenir a violência nas escolas e educar para a paz. Campinas: Verus; 2005.

29. Freire IP, Simão AMV, Ferreira AS. O estudo da violência entre pares no $3^{\circ}$ ciclo do Ensino Básico - questionário aferido para a população escolar portuguesa. RPE 2006; 19(2):157-183.
30. Vizini ST. Evidence of the validation of the Olweus Bullying Questionnaire victim and aggressor version for Brazilian adolescents. [dissertação]. Porto Alegre: Universidade Federal do Rio Grande do Sul; 2017.

31. Reisen A, Viana MC, Santos-Neto ET. Bullying among adolescents: are the victims also perpetrators? Braz J Psychiatry 2019; 41(6):518-529.

32. Santos MHS. Leitura das cidades brasileiras: segregação e espoliação urbanas. REDD 2018; 10(2):23-34.

33. Alves JED, Corrêa S. Igualdade e desigualdade de gênero no Brasil: um panorama preliminar, 15 anos depois do Cairo. In: ABEP, UNFPA, editores. Brasil, 15 anos após a Conferência do Cairo. Campinas: ABEP, UNFPA; 2009. p. 121-231.

34. Boing AF, Melo GR, Boing AC, Moretti-Pires RO, Peres KG, Peres MA. Associação entre depressão e doenças crônicas: um estudo populacional. Rev Saude Publica 2012; 46(4):617-623.

35. Instituto Brasileiro de Geografia e Estatística (IBGE). Pesquisa Nacional por Amostra de Domicílios (PNAD): sintese de indicadores 2015. Brasília: IBGE; 2016.

36. Bonamino A, Alves F, Franco C, Cazelli S. Os efeitos das diferentes formas de capital no desempenho escolar: um estudo à luz de Bourdieu e de Coleman. Rev Bras Educ 2010; 15(45):487-499.

Artigo apresentado em 28/12/2018

Aprovado em 19/09/2019

Versão final apresentada em 21/09/2019

Editores-chefes: Romeu Gomes, Antônio Augusto Moura da Silva 